Published in final edited form as:

Chem Res Toxicol. 2016 February 15; 29(2): 162-168. doi:10.1021/acs.chemrestox.5b00371.

\title{
Identification of New and Distinctive Exposures from Little Cigars
}

\author{
Theodore P. Klupinski $^{*}{ }^{\dagger}$, Erich D. Strozier ${ }^{\dagger}$, David A. Friedenberg ${ }^{\dagger}$, Marielle C. Brinkman ${ }^{\dagger}$, \\ Sydney M. Gordon ${ }^{\dagger}$, and Pamela I. Clark ${ }^{\ddagger}$ \\ †Battelle, 505 King Avenue, Columbus, Ohio 43201, United States \\ FSchool of Public Health, Department of Behavioral and Community Health, Tobacco Center of \\ Regulatory Science, University of Maryland, College Park, Maryland 20742, United States
}

\begin{abstract}
Little cigar mainstream smoke is less well-characterized than cigarette mainstream smoke in terms of chemical composition. This study compared four popular little cigar products against four popular cigarette products to determine compounds that are either unique to or more abundant in little cigars. These compounds are categorized as new or distinctive exposures, respectively. Total particulate matter samples collected from machine-generated mainstream smoke were extracted with methylene chloride, and the extracts were analyzed using two-dimensional gas chromatography-time-of-flight mass spectrometry. The data were evaluated using novel dataprocessing algorithms that account for characteristics specific to the selected analytical technique and variability associated with replicate sample analyses. Among more than 25000 components detected across the complete data set, ambrox was confirmed as a new exposure, and 3methylbutanenitrile and 4-methylimidazole were confirmed as distinctive exposures. Concentrations of these compounds for the little cigar mainstream smoke were estimated at approximately $0.4,0.7$, and $12 \mu \mathrm{g} / \mathrm{rod}$, respectively. In achieving these results, this study has demonstrated the capability of a powerful analytical approach to identify previously uncharacterized tobacco-related exposures from little cigars. The same approach could also be applied to other samples to characterize constituents associated with tobacco product classes or specific tobacco products of interest. Such analyses are critical in identifying tobacco-related exposures that may affect public health.
\end{abstract}

*Corresponding Author: Tel.: 614-424-7595. klupinskit@battelle.org.

Notes

The authors declare no competing financial interest.

ASSOCIATED CONTENT

Supporting Information

The Supporting Information is available free of charge on the ACS Publications website at DOI: 10.1021/acs.chemres-tox.5b00371.

Package information for little cigar products tested; schematic illustration of GC×GC-TOFMS instrument; acquisition parameters for GC $\times$ GC-TOFMS analysis; methods for confirmation analysis; example chromatograms and mass spectra for identified new/distinctive exposures. 


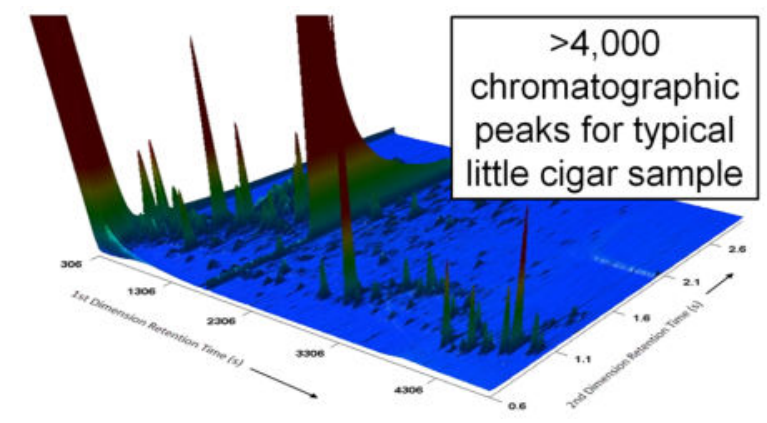

\section{INTRODUCTION}

The objective of this research was to identify new or distinctive chemical exposures associated with little cigars, as a tobacco product class, in contrast to cigarettes. For the purpose of this study, a new exposure is a compound detected in the mainstream smoke from little cigars but not detected in the mainstream smoke from cigarettes, and a distinctive exposure is a compound detected in the mainstream smoke from little cigars at substantially greater concentrations than in the mainstream smoke from cigarettes.

Little cigars, as a product class, may present a distinct concern to regulatory agencies because they exhibit market trends different than those of cigarettes. For example, the use of little cigars in the U.S. increased substantially from 2001 to 2008, even as the total use of combustible tobacco products decreased. ${ }^{1}$ Commercially available little cigars include many flavored products. Characterizing flavors other than menthol were banned in the U.S. for use in cigarettes but not in cigars by the Food and Drug Administration (FDA) in 2009. ${ }^{2}$

Because of this exception, some products that had previously been sold as flavored cigarettes were redesigned as flavored cigars. ${ }^{3}$ Of U.S. students who smoke cigars, $35.9 \%$ reported use of flavored little cigars. ${ }^{4}$

The chemical constituents from little cigars have not been studied to the same extent as those from cigarettes. Mainstream smoke from little cigars may include a mixture of compounds that differs substantially, in both qualitative and quantitative terms, from that of cigarettes. Characterizing the chemical composition of mainstream smoke from little cigars can provide valuable data to the tobacco research community, including regulatory agencies such as the FDA.

More than 6000 compounds have been identified in tobacco smoke. ${ }^{5}$ As different analytical chemistry techniques have been incorporated into common usage over the past several decades, it has become possible to detect more compounds, including those present at tracelevel concentrations. Two-dimensional gas chromatography-time-of-flight mass spectrometry (GC $\times$ GC-TOFMS) is a relatively new analytical technique that has exceptional power for detecting and identifying unknown compounds in complex mixtures such as tobacco smoke. In comparison with one-dimensional gas chromatography techniques, the separation of chemicals by two chromatographic processes greatly improves the ability to isolate individual compounds during analysis. Sample detection by time-of-flight mass spectrometry provides sensitivity approaching that of selective ion monitoring (SIM) for 
quadrupole mass spectrometry and has the advantage of collecting spectra in full-scan mode, thus allowing for nontargeted sample analysis, where tentative compound identities can be assigned according to the mass spectral data. GC $\times$ GC-TOFMS has been applied for the analysis of mainstream smoke samples from cigarettes, including studies on the demonstration of capability, ${ }^{6-8}$ the comparison of commercial cigarettes vs research cigarettes, ${ }^{9}$ and the comparison of cigarettes using two different filter types. ${ }^{10} \mathrm{GC} \times \mathrm{GC}$ TOFMS analysis typically yields complex data sets to which various data-processing approaches can be applied, ${ }^{11-14}$ but it can be challenging to identify the appropriate approach to meet a given technical objective.

The research presented herein describes the use of GC $\times$ GC-TOFMS analysis in combination with efficient data-processing algorithms to identify constituents that differentiate selected tobacco products from a comparison set of tobacco products. In this case, little cigars were compared against cigarettes, a commonly studied product class. Compounds detected in little cigars but not in cigarettes are categorized as new exposures, whereas compounds detected in both product classes but observed at significantly higher concentrations in little cigars are categorized as distinctive exposures. The reported results are important both for the specific information about the chemical composition of mainstream smoke from little cigars and especially for demonstrating the value of this methodology for identifying previously uncharacterized tobacco-related exposures that have the potential to affect public health.

\section{EXPERIMENTAL PROCEDURES}

\section{Product Selection}

Analysis was performed for four little cigar products: Swisher Sweets Original and Swisher Sweets Cherry (Swisher International, Inc.; Jacksonville, FL) and Cheyenne Full Flavor and Cheyenne Menthol (Cheyenne International, LLC; Grover, NC). While four products cannot represent the full range of variability within a product class, these little cigar products were purposely selected for illustrative testing because they hold significant shares of the U.S. market, ${ }^{15,16}$ are from different manufacturers, and include different flavors commonly used in little cigars. In a similar vein, analysis was performed for four cigarette products, Marlboro (Philip Morris USA; Richmond, VA), Newport (Lorillard; Greensboro, NC), and Camel and Pall Mall (RJ Reynolds Tobacco Company; Winston-Salem, NC), that led the U.S. in cigarette market share in $2012^{17}$ and are from different manufacturers. All products were purchased as single lots in Columbus, $\mathrm{OH}$, in July 2014. Package information for the little cigar products is provided in Table S1 (Supporting Information).

\section{Sample Collection}

For each product, four rods (i.e., four separate little cigars or four separate cigarettes) were conditioned according to the ISO/FTC (International Organization for Standardization/ Federal Trade Commission) regimen ${ }^{18}$ in environmental chambers (Webber models AF16-40+350F, AF16-100+350, or AF16-100+350H; Webber Manufacturing Company, Indianapolis, IN). The rods were then smoked based on the ISO/FTC smoking regimen ${ }^{18,19}$ in separate ports of a linear five-port smoking machine (Hawktech FP2000, Tri-City 
Machine Works). Total particulate matter (TPM) samples were collected from the mainstream smoke on Cambridge glass fiber filters (one rod/pad). TPM masses were determined using an analytical balance, ${ }^{20,21}$ and the TPM samples were extracted and analyzed according to the procedures described below. The mass lost during combustion was determined by the difference between the mass of the original rod and the mass of the rod after it was smoked, where both were determined using an analytical balance. Blank runs were performed by operating the smoking machine similarly but with no tobacco product in a given port. Filters were collected from the blank runs and treated identically to filters collected from the tobacco product runs.

\section{Chemical Analysis}

Many reported methods for the extraction of mainstream smoke TPM are designed for specific classes of compounds. For example, the nonpolar solvent cyclohexane is used to extract the nonpolar compound benzo[a]pyrene, ${ }^{22}$ and the polar solvent methanol is used to extract the polar nitrosamine compounds. ${ }^{23}$ While there is no general approach to effectively extract unknown chemicals from a complex matrix such as TPM, methylene chloride was selected for this study because its moderate polarity is suitable for extracting a wide range of compounds of interest. No other extraction solvents were tested. Tobacco smoke constituents that may not have been extracted into methylene chloride include highly polar compounds such as carboxylic acids, which are typically not amenable to gas chromatography (GC) analysis and thus would not have been detected even if they were present in the extracts.

Each TPM-containing filter was extracted using three $5 \mathrm{~mL}$ aliquots of methylene chloride by gently swirling the solvent about the filter in a $60 \mathrm{~mL}$ glass jar. The combined extracts from a single TPM sample had a total volume of about $12-13 \mathrm{~mL}$ due to solvent loss to evaporation and absorption into the filter. A portion of each combined methylene chloride extract was concentrated 3-fold by evaporation under a gentle stream of nitrogen.

A $100 \mu \mathrm{L}$ aliquot of each concentrated extract was spiked with a stock solution of an internal standard, acenaphthene- $d_{10}$, to yield a concentration of $200 \mathrm{ng} / \mathrm{mL}$. Acenaphthene- $d_{10}$ was selected as an internal standard because its retention time falls close to the middle of the time range monitored in the chromatogram and it has a distinct mass spectrum from that of the polycyclic aromatic hydrocarbon acenaphthene, which could potentially be present in any of the TPM extracts. The spiked, concentrated extracts were then analyzed using a Leco Pegasus 4D GC $\times$ GC-TOFMS (Leco; St. Joseph, MI). This instrument utilizes an Agilent 7890 GC (Agilent; San Mateo, CA) fitted with a cryogenically cooled two-stage modulator and a secondary temperature-programmable oven mounted inside the main GC oven. The GC is coupled to a Leco Pegasus time-of-flight mass spectrometer. Chromatographic columns were selected to separate compounds by boiling point in the first dimension and by polarity in the second dimension. A schematic illustration of a GC×GC-TOFMS instrument is provided in Figure S1, and acquisition parameters are provided in Table S2 (Supporting Information).

Authentic standards of ambrox (purchased as enantiomerically pure (-)-ambroxide; purity = $99.9 \%$ by GC, per supplier), 3-methylbutanenitrile (purity $=99.6 \%$ by GC, per supplier), 
and 4-methylimidazole (purity $=98.4 \%$ by $\mathrm{HCl}$ titration, per supplier) were obtained from Sigma-Aldrich (St. Louis, MO) to prepare standards for confirmation analysis.

\section{Data Evaluation}

The data set consists of 16 little cigar samples (4 products $\times 4$ rods/product), 16 cigarette samples ( 4 products $\times 4$ rods/product), and 8 blank samples. Data acquisition, peak-finding, and spectral deconvolution were performed using Leco ChromaTOF v4.50 software. Preliminary component identifications were performed by automated matching of deconvoluted component mass spectra with the National Institute of Standards and Technology (NIST) 11 Mass Spectral Library. ${ }^{24}$

Peak Tables-For each of the 40 samples, a peak table was generated showing the retention time, response (peak height), and Chemical Abstract Services registry number (CASRN) of the best mass spectral library match. Due to the large amount of data acquired, manual inspection of the mass spectra and verification of assigned component identities were not feasible; however, regardless of whether the components are identified correctly at this stage of the process, the assigned CASRNs are useful tags to allow for cross-comparison of multiple samples. The peak tables were then filtered to remove known analysis system artifacts such as column siloxane bleed and injection solvent. After filtering, a single peak table included several hundred to several thousand component peaks. The filtered peak tables were used as input to the software algorithms.

Software Algorithms-Novel data-processing algorithms were used to determine new or distinctive exposure candidates. These algorithms were designed to account for the large data set, characteristics specific to GC $\times$ GC-TOFMS analysis, and variability associated with replicate sample analyses.

Responses for compounds at high concentrations sometimes span much of the seconddimension retention-time range (only $3 \mathrm{~s}$ in total), leading to the presence of multiple peaks for a single compound, whereas the first-dimension retention time is generally accurate to within 6 to $9 \mathrm{~s}$. To accommodate this analytical variability, the base detection for a given CASRN was assigned to the retention time pair corresponding to the largest peak.

Additional peaks for the same CASRN that were within $6 \mathrm{~s}$ of the base first-dimension retention time and that exhibited any second-dimension retention time were merged into the base peak by summing the peak heights. The summed height was then normalized by dividing by the internal standard peak height for that sample. Thus, responses for all peaks associated with a given CASRN that lie within a rectangle $12 \mathrm{~s}$ wide by $3 \mathrm{~s}$ tall in chromatographic space were evaluated as one entity. In practice, the distribution of data points within this rectangle often has a roughly oval shape, and the approach, designated the oval height method, is useful in controlling for potential data artifacts.

For any CASRN observed across the entire data set, every sample was evaluated to determine whether a peak for that CASRN was detected and, if so, what the oval height was. From this information, three subsets of CASRNs were identified, as follows: 
1. New exposure candidate: a given CASRN is present in all little cigar samples, absent from all cigarette samples, and absent from all blank samples.

2. Class one distinctive exposure candidate: a given CASRN has peaks with larger oval heights in any little cigar sample in comparison with any cigarette sample.

3. Class two distinctive exposure candidate: a given CASRN has peaks with a significantly larger oval height in little cigar samples in comparison with cigarette samples, as determined by a value of Cohen's effect size ${ }^{25}$ that is $>0.6$, a common threshold for moderate differentiation between two sets of samples.

$$
\text { Cohen's effect size }=d=\frac{\bar{x}_{1}-\overline{x_{2}}}{s}
$$

where

$$
\begin{aligned}
& s=\sqrt{\frac{\left(n_{1}-1\right) s_{1}^{2}+\left(n_{2}-1\right) s_{2}^{2}}{n_{1}+n_{2}-2}} \\
& x_{i}=\text { observed mean from } n_{i} \text { samples in set } i \\
& s_{i}=\text { observed standard deviation from } n_{i} \text { samples in set } i
\end{aligned}
$$

To minimize the potential for reporting false-positive results, use of the data-processing algorithms was supplemented for compounds of interest by technical reviews of the chromatography data and the mass spectral data and, in some cases, confirmation analyses, as described below.

Assessment of Mass Spectra-For all new exposure candidates and selected distinctive exposure candidates, the mass spectra recorded for the samples were first compared with the NIST 11 Mass Spectral Library for tentative identity assignment and then evaluated for consistency in each sample for which a detection was indicated for the same CASRN. In some cases, the CASRN assigned by automated library matching was considered to be inaccurate (e.g., due to a poor match to the library mass spectrum or peaks for the assigned compound showing inconsistent retention times in different samples). In other cases, the CASRN assigned by automated library matching was considered to be accurate, indicating a tentative identification of the new/distinctive exposure candidate.

\section{Confirmation Analysis}

In experiments to confirm or reject the presence of selected tentatively identified new or distinctive exposure candidates in the samples, aliquots of some concentrated extracts were later spiked with authentic standards of the relevant compounds and analyzed by either GC $\times$ GC-TOFMS or liquid chromatography-tandem mass spectrometry (LC-MS/MS). Details are provided in the Supporting Information. 


\section{RESULTS}

On average, the little cigar products, in contrast to the cigarette products, had total masses that were $\sim 45 \%$ greater, lost $\sim 35 \%$ more mass during combustion, and yielded $\sim 40 \%$ larger amounts of TPM, as illustrated in Table 1. All differences are statistically significant at the 95\% confidence level.

Analysis of a given little cigar product yielded $\sim 2800$ to $\sim 5700$ peaks in the peak table, whereas analysis of a given cigarette product yielded $\sim 1800$ to $\sim 3800$ peaks (Figure 1). The median value for little cigars is significantly greater than that for cigarettes at the $95 \%$ confidence level according to the Mann-Whitney $\mathrm{U}$ test,${ }^{26}$ which was applied due to the apparent non-normal distribution of the little cigars data. The large number of detected components for all tobacco products reflects both the sensitivity of GC $\times$ GC-TOFMS analysis and the complexity of mainstream smoke. Illustrative examples of chromatograms from little cigar and cigarette samples are provided in Figure 2.

The data from all 40 samples included more than 25000 components, marked by distinct CASRNs. Data evaluation using the software algorithms described in the Experimental Procedures led to the identification of one new exposure candidate, 36 class one distinctive exposure candidates, and 3276 class two distinctive exposure candidates, as defined previously. It would be impractical to evaluate the analytical data for such a large number of components. Therefore, an approach to prioritization was developed, and mass spectra were assessed by comparison with the NIST 11 Mass Spectral Library for the following CASRNs:

- $\quad$ the new exposure candidate,

- $\quad$ all class one distinctive exposure candidates,

- $\quad$ several class two distinctive exposure candidates characterized by large effect sizes, and

- $\quad$ several class two distinctive exposure candidates characterized by large values for the ratio of the mean oval height from little cigar samples to the mean oval height from cigarette samples.

Following the assessments of the mass spectra, numerous CASRNs were discounted from further consideration for reasons such as exhibiting poor matches to the library mass spectra or inconsistent retention times across different samples. 3-Methylbutanenitrile was designated as a second possible new exposure after it had initially been a class one distinctive exposure candidate because a peak for a single cigarette sample that was given a preliminary assignment as 3-methylbutanenitrile was discounted during the assessments of the mass spectra. Of the CASRNs that were considered as tentative identifications, three of particular interest were selected for investigation by confirmation analysis, as follows:

- $\quad$ ambrox (CASRN 100679-85-4), a possible new exposure,

- 3-methylbutanenitrile (CASRN 625-28-5), a possible new exposure, and

- $\quad$ 4-methylimidazole (CASRN 822-36-6), a possible distinctive exposure. 
Illustrations of the analytical data supporting the detections of all three compounds are provided in Figures S2-S4 (Supporting Information). There were other distinctive exposure candidates considered as tentative identifications, suggesting that there could be additional distinctive exposures beyond those discussed below, but they were not carried on for confirmation analysis for this focused study on capability demonstration.

Ambrox and 3-methylbutanenitrile were confirmed as positive identifications in little cigar samples by separate $\mathrm{GC} \times \mathrm{GC}$-TOFMS analyses of concentrated extracts (one per product) concurrent with concentrated extracts that had been spiked with the authentic compounds. Analyses of additional spiked samples demonstrated that ambrox and 3-methylbutanenitrile were not present at detectable levels in cigarette samples (one per product) and two blank samples. Therefore, ambrox and 3-methylbutanenitrile meet the criteria for new exposures as defined for this study.

4-Methylimidazole was confirmed as a positive identification in both little cigar and cigarette samples by LC-MS/MS analyses of concentrated extracts (one per product) concurrent with concentrated extracts that had been spiked with the authentic compounds. Further analyses indicated that 4-methylimidazole was not present at detectable levels in two blank samples.

The identities, CASRNs, molecular formulas, and estimated concentrations of the new and distinctive exposures are indicated in Table 2. The estimated concentrations were interpolated by comparing the peak response (height for GC×GC-TOFMS analysis or area for LC-MS/MS analysis) for a native sample to the increase in peak response for the corresponding spiked sample. These concentrations are regarded as semiquantitative estimates, generally reported to one significant figure, because the analytical method was not evaluated for quantitative accuracy and precision. Ambrox and 3-methylbutanenitrile are estimated to be present at $<1 \mu \mathrm{g} / \mathrm{rod}$ each in the mainstream smoke from little cigars. These low concentrations, detected from the nontargeted analysis of TPM for a single little cigar, demonstrate the sensitivity of GC $\times$ GC-TOFMS. 4-Methylimidazole is designated as a distinctive exposure because the estimated concentrations are significantly greater in little cigars than in cigarettes, even when normalized to the observed TPM masses.

\section{DISCUSSION}

\section{Compound Characteristics}

Characteristics of the three new and distinctive exposures are discussed briefly in the following paragraphs. It is noted that any compound designated as a new exposure could, in fact, be present in one or more of the cigarette products at a concentration lower than the detection limit of the analytical method.

Ambrox, which is used as a fragrance ingredient in perfumes, has an "amber, woody, mossy scent". ${ }^{27}$ (CASRN 100679-85-4, indicating relative stereochemistry for ambrox, is used for identification purposes in the NIST 11 Mass Spectral Library. In some sources, ambrox may instead be identified using either CASRN 6790-58-5, indicating an enantiomerically pure material, or CASRN 3738-00-9, indicating that stereochemistry is not specified.) In a 
tobacco industry study, ambrox was detected in essential oil from Turkish tobacco and was considered to have flavor potential. ${ }^{28}$ No references were found indicating the presence of ambrox in tobacco smoke. Thus, the detection of ambrox in little cigar mainstream smoke appears to be novel.

3-Methylbutanenitrile has been reported as a constituent of tobacco smoke, along with other aliphatic nitriles, for example, pentanenitrile $\left(\mathrm{C}_{5} \mathrm{H}_{9} \mathrm{~N}\right)$ and 2-methylpropanenitrile $\left(\mathrm{C}_{4} \mathrm{H}_{7} \mathrm{~N}\right) .{ }^{29} \mathrm{In}$ a tobacco industry study, 3-methylbutanenitrile was detected in the mainstream smoke for four out of five cigarette products tested, with concentrations of up to $0.2 \mu \mathrm{g} / \mathrm{rod} .{ }^{30} 3$-Methylbutanenitrile was first categorized as a new exposure because it was not detected for the cigarettes tested in the present study, in which the estimated detection limit is $0.6 \mu \mathrm{g} / \mathrm{rod}$ (see Table 2 ). In a broader context that considers previously reported results for tobacco constituents, it is designated as a distinctive exposure.

In general, the toxicity of nitriles is considered to be low; however, aliphatic nitriles can be metabolized to liberate cyanide ions in vivo, ${ }^{31}$ raising concerns about their potential toxicity. 3-Methylbutanenitrile is reported to have an oral $\mathrm{LD}_{50}$ of $233 \mathrm{mg} / \mathrm{kg}$ in mice, and signs of peripheral nervous system and cardiovascular toxicity were noted in frogs and rats at much higher subcutaneous doses. ${ }^{32}$ Overall, the toxicity of 3-methylbutanenitrile is not wellcharacterized, particularly via the inhalation route.

4-Methylimidazole, which has been previously reported as a constituent of tobacco smoke from cigarettes, ${ }^{33}$ has been examined in numerous toxicity and carcinogenicity investigations. ${ }^{34,35}$ In an ingestion study using mice, it was associated with increased incidences of alveolar/bronchiolar adenoma and carcinoma. ${ }^{36}$ 4-Methylimidazole is included on California's Proposition 65 list of substances that can cause cancer. ${ }^{37}$ This designation has led some beverage companies to change their manufacturing processes in order to decrease the amount of 4-methylimidazole found in commercially sold colas. ${ }^{38}$

\section{CONCLUSIONS}

This study has demonstrated the use of GC×GC-TOFMS analysis, supported by efficient data-processing algorithms, to identify previously uncharacterized tobacco-related exposures from little cigars. GC $\times \mathrm{GC}$-TOFMS can be applied for the nontargeted detection of numerous compounds from complex samples with high sensitivity (e.g., detections at estimated concentration $<1 \mu \mathrm{g} / \mathrm{rod}$ ), high selectivity (e.g., over 5000 distinct peaks can be detected from a single sample), and the ability to assign tentative compound identities according to the mass spectral data. Mainstream smoke from four popular little cigar products (Swisher Sweets Original, Swisher Sweets Cherry, Cheyenne Full Flavor, and Cheyenne Menthol) was characterized in contrast to four popular cigarette products (Marlboro, Newport, Camel, and Pall Mall) by the presence of ambrox and 3methylbutanenitrile as new exposures and 4-methylimidazole as a distinctive exposure. In light of previously published results for tobacco constituents, 3-methylbutanenitrile was ultimately categorized instead as a distinctive exposure. With regard to characterizing little cigar mainstream smoke, additional research could be conducted either to evaluate the data set further to identify potentially more new or distinctive exposures or to collect additional 
data from an expanded selection of little cigar products to establish the robustness of any new or distinctive exposure. More generally, this analytical approach can be applied for comparisons of other sample data to characterize constituents associated with tobacco product classes or specific tobacco products of interest. Such analyses are critical in identifying tobacco-related exposures that may affect public health.

\section{Supplementary Material}

Refer to Web version on PubMed Central for supplementary material.

\section{Acknowledgments}

Funding

This work was supported by the National Institutes of Health (NIH) and the Food and Drug Administration (FDA) Center for Tobacco Products (CTP) (grant number 1P50CA180523). The content is solely the responsibility of the authors and does not necessarily represent the official views of the NIH or the FDA.

The authors thank Stephanie Makselan, Stephanie Buehler, Courtney Granville, Derik Heiss, Hyoshin Kim, Robyn Kroeger, Jill Priebe, Betsy Thompson, Cheryl Triplett, Jim Watts, and Laura Wilson of Battelle and Blair Coleman of the FDA CTP for their contributions to this study.

\section{ABBREVIATIONS}

CASRN Chemical Abstract Services registry number

CTP

FDA

FTC

GC $\times$ GC-TOFMS

ISO

LC-MS/MS

NIH

NIST

SIM

TPM
Center for Tobacco Products

Food and Drug Administration

Federal Trade Commission

two-dimensional gas chromatography-time-of-flight mass spectrometry

International Organization for Standardization

liquid chromatography-tandem mass spectrometry

National Institutes of Health

National Institute of Standards and Technology

selective ion monitoring

total particulate matter

\section{References}

1. United States Centers for Disease Control and Prevention. Consumption of cigarettes and combustible tobacco - United States, 2000-2011. Morb Mortal Wkly Rep. 2012; 61:565-569.

2. Guidance for Industry and FDA Staff: General Questions and Answers on the Ban of Cigarettes that Contain Certain Characterizing Flavors. 2. United States Department of Health and Human Services, Food and Drug Administration, Center for Tobacco Products; Silver Spring, MD: 2009. 
http://www.fda.gov/downloads/TobaccoProducts/ProtectingKidsfromTobacco/FlavoredTobacco/ UCM195420.pdf [accessed April 19, 2014]

3. Preventing Tobacco Use Among Youth and Young Adults: A Report of the Surgeon General. United States Department of Health and Human Services, Public Health Service, Office of the Surgeon General; Rockville, MD: 2012. http://www.surgeongeneral.gov/library/reports/preventing-youthtobacco-use/full-report.pdf [accessed August 20, 2015]

4. King BA, Tynan MA, Dube SR, Arrazola R. Flavored-little-cigar and flavored-cigarette use among U.S. middle and high school students. J Adolesc Health. 2014; 54:40-46. [PubMed: 24161587]

5. Rodgman, A.; Perfetti, TA. The Chemical Components of Tobacco and Tobacco Smoke. 2. CRC Press; Boca Raton, FL: 2013.

6. Dallüge J, van Stee LLP, Xu X, Williams J, Beens J, Vreuls RJJ, Brinkman UATh. Unravelling the composition of very complex samples by comprehensive gas chromatography coupled to time-offlight mass spectrometry cigarette smoke. J Chromatogr A. 2002; 974:169-184. [PubMed: 12458936]

7. Lu X, Cai J, Kong H, Wu M, Hua R, Zhao M, Liu J, Xu G. Analysis of cigarette smoke condensates by comprehensive two-dimensional gas chromatography/time-of-flight mass spectrometry I acidic fraction. Anal Chem. 2003; 75:4441-4451. [PubMed: 14632048]

8. Lu X, Zhao M, Kong H, Cai J, Wu J, Wu M, Hua R, Liu J, Xu G. Characterization of complex hydrocarbons in cigarette smoke condensate by gas chromatography-mass spectrometry and comprehensive two-dimensional gas chromatography-time-of-flight mass spectrometry. $\mathrm{J}$ Chromatogr A. 2004; 1043:265-273. [PubMed: 15330101]

9. Gröger T, Welthagen W, Mitschke S, Schäffer M, Zimmermann R. Application of comprehensive two-dimensional gas chromatography mass spectrometry and different types of data analysis for the investigation of cigarette particulate matter. J Sep Sci. 2008; 31:3366-3374. [PubMed: 18925627]

10. Brokl M, Bishop L, Wright CG, Liu C, McAdam K, Focant JF. Multivariate analysis of mainstream tobacco smoke particulate phase by headspace solid-phase micro extraction coupled with comprehensive two-dimensional gas chromatography-time-of-flight mass spectrometry. $\mathbf{J}$ Chromatogr A. 2014; 1370:216-229. [PubMed: 25454146]

11. Zeng ZD, Hugel HM, Marriott PJ. Chemometrics in comprehensive multidimensional separations. Anal Bioanal Chem. 2011; 401:2373-2386. [PubMed: 21660415]

12. Reichenbach SE, Tian X, Cordero C, Tao Q. Features for non-targeted cross-sample analysis with comprehensive two-dimensional chromatography. J Chromatogr A. 2012; 1226:140-148. [PubMed: 21855071]

13. Pierce KM, Kehimkar B, Marney LC, Hoggard JC, Synovec RE. Review of chemometric analysis techniques for comprehensive two dimensional separations data. J Chromatogr A. 2012; 1255:311. [PubMed: 22727556]

14. Murray JA. Qualitative and quantitative approaches in comprehensive two-dimensional gas chromatography. J Chromatogr A. 2012; 1261:58-68. [PubMed: 22647189]

15. Tobacco Cigars. [accessed July 10, 2014] CSP Category Management Handbook. Apr. 2013 http:// www.cspnet.com/sites/default/files/magazine-files/CMH13_Tobacco_Cigars.pdf

16. Tobacco Cigars. [accessed July 10, 2014] CSP Category Management Handbook. Apr. 2014 http:// www.cspnet.com/sites/default/files/magazine-files/Tobacco_Cigars_CMH_2014.pdf

17. Tobacco Brand Preferences. United States Centers for Disease Control and Prevention, National Center for Chronic Disease Prevention and Health Promotion, Office on Smoking and Health; Atlanta, GA: http://www.cdc.gov/tobacco/data_statistics/fact_sheets/tobacco_industry/ brand_preference/ [accessed July 9, 2014]

18. Tobacco and Tobacco Products - Atmosphere for Conditioning and Testing. 4. International Organization for Standardization; Geneva, Switzerland: 1999. ISO 3402:1999(E)

19. Routine Analytical Cigarette-Smoking Machine — Definitions and Standard Conditions. 4. International Organization for Standardization; Geneva, Switzerland: 2000. ISO 3308:2000(E)

20. Cigarettes - Determination of Total and Nicotine-Free Dry Particulate Matter Using a Routine Analytical Smoking Machine. 3. International Organization for Standardization; Geneva, Switzerland: 2000. ISO 4387:2000(E) 
21. Cigarettes - Determination of Total and Nicotine-Free Dry Particulate Matter Using a Routine Analytical Smoking Machine. 3. International Organization for Standardization; Geneva, Switzerland: 2008. Amendment 1, ISO 4387:2000/Amd.1:2008(E)

22. Method - Determination of Benzo(a)pyrene in Mainstream Smoke. British American Tobacco p.l.c; London: 2008. http://www.bat.com/groupms/sites/bat_9gvjxs.nsf/vwPagesWebLive/DO7AXGCL/ \$FILE/medMD7DAE8Z.pdf [accessed January 11, 2015]

23. Method - Determination of Tobacco-Specific Nitrosamines in Mainstream Smoke. British American Tobacco p.1.c; London: 2008. http:/www.bat.com/groupms/sites/bat_9gvjxs.nsf/ vwPagesWebLive/DO7AXGCL/\$FILE/medMD7DAEBN.pdf [accessed January 11, 2015]

24. NIST/EPA/NIH Mass Spectral Database (NIST 11) and NIST Mass Spectral Search Program, version 2.0g. United States Department of Commerce, National Institute of Standards and Technology; Gaithersburg, MD: 2011.

25. Cohen J. A power primer. Psychol Bull. 1992; 112:155-159. [PubMed: 19565683]

26. Hollander, M.; Wolfe, DA. Nonparametric Statistical Methods. John Wiley \& Sons; New York: 1973. p. 27-75.

27. Ash, M.; Ash, I. Handbook of Flavors and Fragrances. Synapse Information Resources, Inc; Endicott, NY: 2008.

28. Turkish Tobacco Essential Oil. Truth Tobacco Industry Documents RDM. 1978; 27 [accessed January 8, 2016] http://industrydocuments.library.ucsf.edu/tobacco/docs/llkn0096.

29. Schmeltz I, Hoffmann D. Nitrogen-containing compounds in tobacco and tobacco smoke. Chem Rev. 1977; 77:295-311.

30. Comparative smoke studies. VI. Winston/Vantage/Now/Marlboro/Merit. Truth Tobacco Industry Documents RDR. 1977; 4 [accessed January 8, 2016] http://industrydocuments.library.ucsf.edu/ tobacco/docs/ypxv0094.

31. Silver EH, Kuttab SH, Hasan T, Hassan M. Structural considerations in the metabolism of nitriles to cyanide in vivo. Drug Metab Dispos. 1982; 10:495-498. [PubMed: 6128199]

32. Isovaleronitrile. ChemIDplus. United States Department of Health and Human Services, National Institutes of Health, National Library of Medicine; Bethesda, MD: http://chem.sis.nlm.nih.gov/ chemidplus/chemidlite.jsp [accessed December 10, 2014]

33. Schumacher JN, Green CR, Best FW, Newell MP. Smoke composition. An extensive investigation of the water-soluble portion of cigarette smoke. J Agric Food Chem. 1977; 25:310-320. [PubMed: 838966]

34. NTP Technical Report on the Toxicology and Carcinogenesis Studies of 4-Methylimidazole (CAS No. 822-36-6) in F344/N Rats and B6C3F 1 Mice (Feed Studies), NIH Publication No. 07-4771, United States Department of Health and Human Services, Public Health Service, National Institutes of Health, National Toxicology Program, Research Triangle Park, NC, 2007.

35. Hengel M, Shibamoto T. Carcinogenic 4(5)-methylimidazole found in beverages, sauces, and caramel colors: chemical properties, analysis, and biological activities. J Agric Food Chem. 2013; 61:780-789. [PubMed: 23294412]

36. Chan PC, Hills GD, Kissling GE, Nyska A. Toxicity and carcinogenicity studies of 4methylimidazole in F344/N rats and B6C3F1 mice. Arch Toxicol. 2008; 82:45-53. [PubMed: 17619857]

37. 4-Methylimidazole (4-MEI) A Fact Sheet. California Environmental Protection Agency, Office of Environmental Health Hazard Assessment; Sacramento, CA: 2012. http://oehha.ca.gov/ public_info/facts/4MEIfacts_021012.html [accessed August 30, 2015]

38. Geller, M. [accessed December 11, 2014] Coke, Pepsi make changes to avoid cancer warning. Reuters. Mar 9. 2012 http://www.reuters.com/article/2012/03/09/us-coke-pepsiidUSBRE82814V20120309 


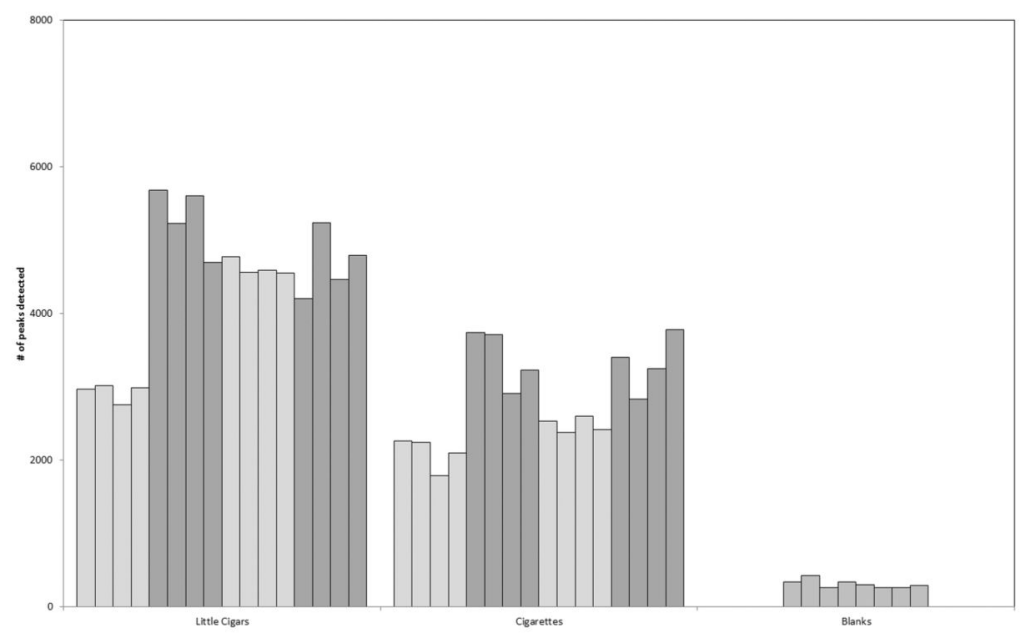

Figure 1.

Numbers of peaks in peak tables for little cigar, cigarette, and blank samples. Little cigar samples are marked by bars representing four replicates for each of (from left to right) Cheyenne Menthol, Swisher Sweets Cherry, Swisher Sweets Original, and Cheyenne Full Flavor. Cigarette samples are marked by bars representing four replicates for each of (from left to right) Marlboro, Newport, Pall Mall, and Camel. 
Little Cigar: Swisher Sweets Original

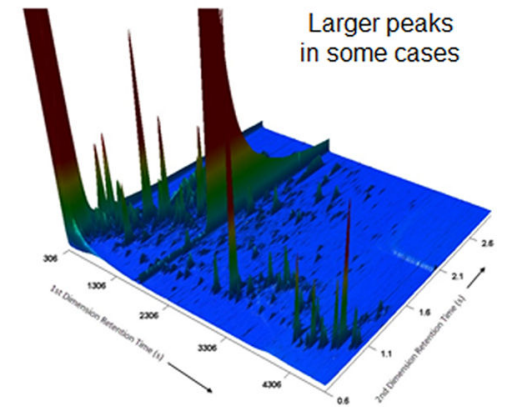

More numerous peaks: 4,562 Peaks

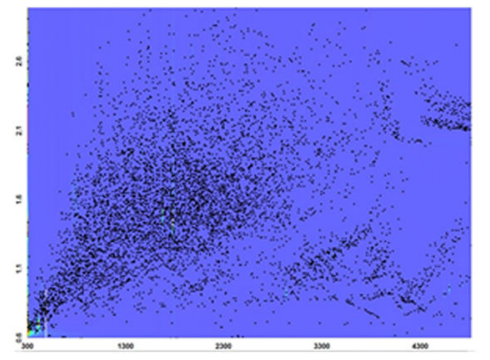

Cigarette: Camel

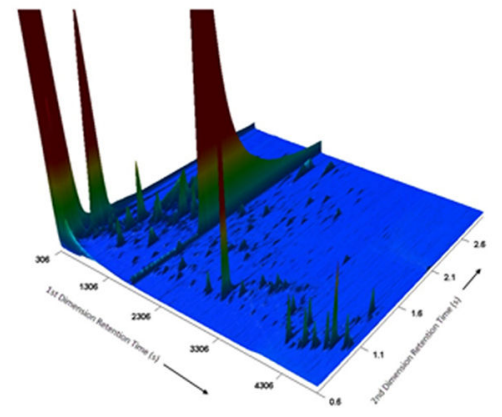

2,832 Peaks

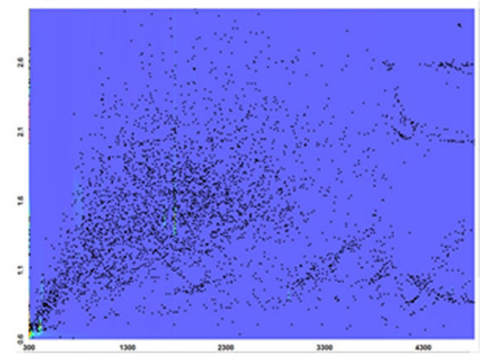

Figure 2.

Example GCXGC-TOFMS chromatograms for little cigar and cigarette samples. Top: Threedimensional chromatograms, where $x$ and $y$ axes show retention times on first and second chromatographic columns, respectively, and $z$ axis indicates combined responses from detected ions in mass spectra. Bottom: Two-dimensional chromatograms, where $x$ and $y$ axes show retention times on first and second chromatographic columns, respectively, and each dot indicates a peak, regardless of response. 


\section{Table 1}

\section{Relevant Masses Observed for Two Tobacco Product Classes}

\begin{tabular}{|c|c|c|}
\hline & little cigars $(n=16)$ & cigarettes $(n=16)$ \\
\hline mass of $\operatorname{rod}^{a}(\mathrm{~g})$ & $1.35^{b}(0.086)$ & $0.93(0.036)$ \\
\hline mass lost during combustion ${ }^{a}(\mathrm{~g})$ & $0.88^{b}(0.067)$ & $0.65(0.060)$ \\
\hline mass of TPM ${ }^{a}(\mathrm{mg})$ & $24^{b}(4.2)$ & $17(7.2)$ \\
\hline
\end{tabular}

a Mean values, with standard deviations indicated in parentheses.

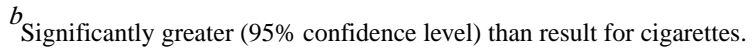




\section{롤 \\ 골}

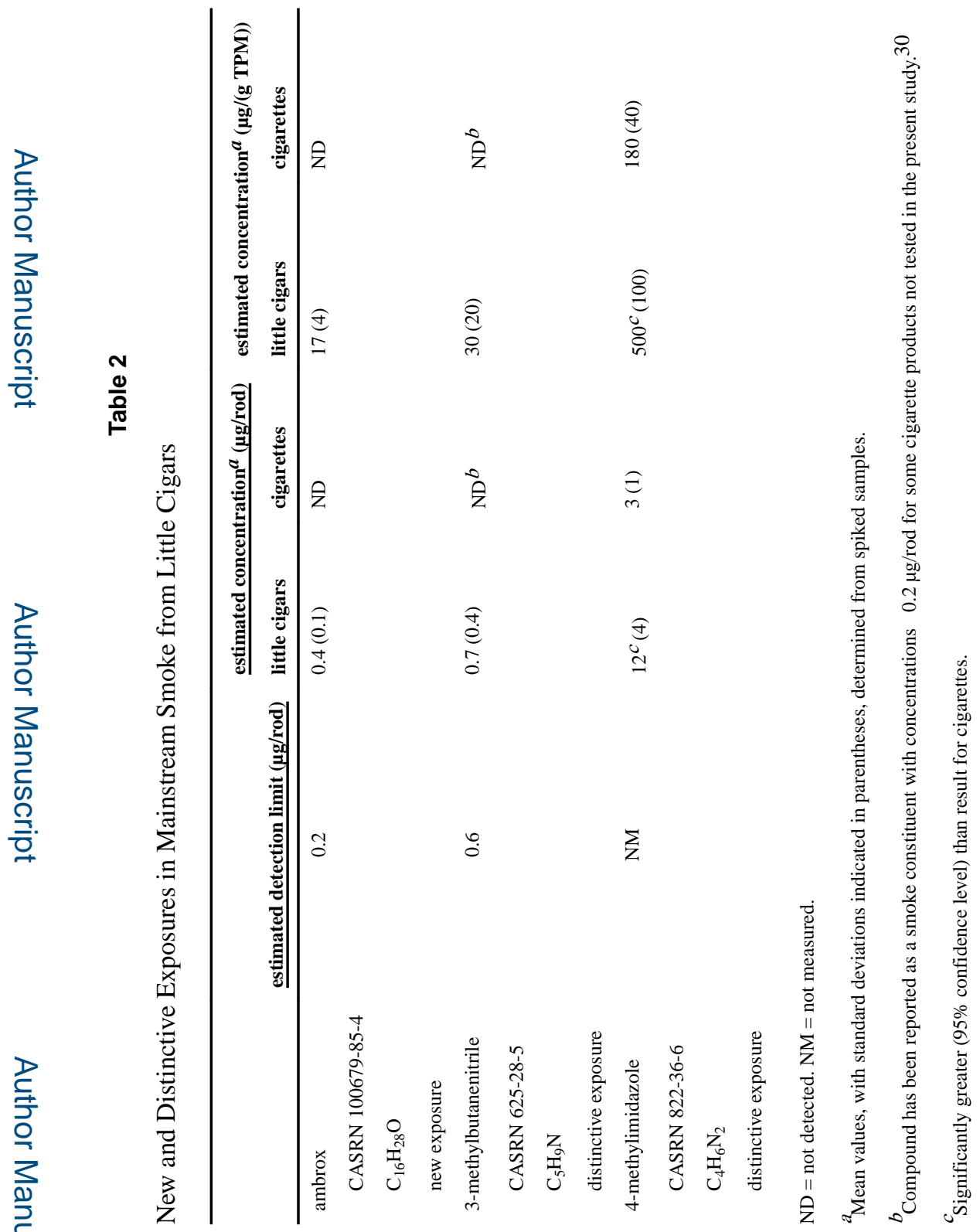

Chem Res Toxicol. Author manuscript; available in PMC 2017 February 15. 